\title{
X-ray Phase-Contrast Imaging and Metrology through Unified Modulated Pattern Analysis
}

\author{
Marie-Christine Zdora, ${ }^{1,2}{ }^{*}$ Pierre Thibault, ${ }^{3}$ Tunhe Zhou, ${ }^{4, \dagger}$ Frieder J. Koch, ${ }^{5}$ Jenny Romell, ${ }^{4}$ \\ Simone Sala, ${ }^{1,2}$ Arndt Last, ${ }^{5}$ Christoph Rau, ${ }^{1,6,7}$ and Irene Zanette ${ }^{1}$ \\ ${ }^{1}$ Diamond Light Source, Harwell Science and Innovation Campus, Didcot, Oxfordshire OX11 ODE, United Kingdom \\ ${ }^{2}$ Department of Physics \& Astronomy, University College London, London WC1E 6BT, United Kingdom \\ ${ }^{3}$ Department of Physics \& Astronomy, University of Southampton, Southampton SO17 1BJ, United Kingdom \\ ${ }^{4}$ Department of Applied Physics, Royal Institute of Technology, 10691 Stockholm, Sweden \\ ${ }^{5}$ Institute of Microstructure Technology, Karlsruhe Institute of Technology, 76344 Eggenstein-Leopoldshafen, Germany \\ ${ }^{6}$ School of Materials, University of Manchester, Manchester M1 7HS, United Kingdom \\ ${ }^{7}$ Department of Otolaryngology, Northwestern University, Feinberg School of Medicine, Chicago, Illinois 60611, USA
}

(Received 13 November 2016; published 18 May 2017)

\begin{abstract}
We present a method for X-ray phase-contrast imaging and metrology applications based on the sampleinduced modulation and subsequent computational demodulation of a random or periodic reference interference pattern. The proposed unified modulated pattern analysis (UMPA) technique is a versatile approach and allows tuning of signal sensitivity, spatial resolution, and scan time. We characterize the method and demonstrate its potential for high-sensitivity, quantitative phase imaging, and metrology to overcome the limitations of existing methods.
\end{abstract}

DOI: 10.1103/PhysRevLett.118.203903

In the last decades phase-contrast imaging and phase sensing have found numerous applications in a broad range of fields from the visible light regime to the $\mathrm{x}$-ray and electron domains. Improving computing resources and algorithms have changed profoundly the way measurements are made in these fields, pushing the trend to take multiple complementary measurements to disentangle a posteriori the useful information from the instrument response. Approaches based on this principle were used early on for electron microscopy [1] and more recently for a range of applications such as laser wave front sensing [2], phase sensing and imaging through in-line holography and ptychography in the visible light $[3,4]$ and the $x$-ray regime [5-9], and even as a means to align space telescopes using near-infrared radiation [10].

The same evolution can be found in x-ray grating-based imaging (GBI) [11-13] and, more recently, speckle-based imaging (SBI) [14-16]. Both of these full-field phasecontrast techniques rely on analyzing the sample-induced changes to a near-field interference pattern created by a phase modulator to retrieve the differential phase shift, transmission, and small-angle scattering signals of the specimen [16-19]. GBI and SBI can both be operated under conditions of limited coherence of the X-ray beam and have been successfully translated from synchrotrons to

Published by the American Physical Society under the terms of the Creative Commons Attribution 4.0 International license. Further distribution of this work must maintain attribution to the author(s) and the published article's title, journal citation, and DOI. polychromatic laboratory sources [20-22]. Their implementations in the tomographic mode make them suitable candidates for biomedical applications and material characterization, among others [23-29].

Both GBI and SBI were initially used in the single-shot mode, through moiré pattern analysis [12,30] and realspace speckle-tracking cross-correlation analysis [14,15]. Analysis on single frames allows for short acquisition times, but suffers from limited spatial resolution, as areas covering several pixels are required to extract useful information. A second mode of operation, also commonly used for both techniques, involves scanning the reference pattern in equidistant subfeature steps. This approach is known as the phase-stepping [23,31] and speckle-scanning $[16,32]$ modes and allows for a pixel-wise reconstruction to be performed, thus improving resolution substantially.

Despite ongoing efforts to improve these techniques [32-35], important drawbacks still impede their widespread implementation. GBI stepping analysis assumes that the interference pattern is sinusoidal and undistorted in amplitude and period. The step sizes are often as small as $100 \mathrm{~nm}$. The common implementation of GBI with two gratings is cumbersome, sensitive to instabilities, and dose inefficient. For SBI, the scanning mode involves the difficult task of acquiring several hundreds of frames taken with step sizes of tens of nanometers.

Here we propose a single solution for the current limitations of both GBI and SBI, which will facilitate their wider implementation, even under suboptimal experimental conditions, while preserving the achievable image quality. The method, called "unified modulated pattern analysis" (UMPA), is applicable to any type of reference pattern, 


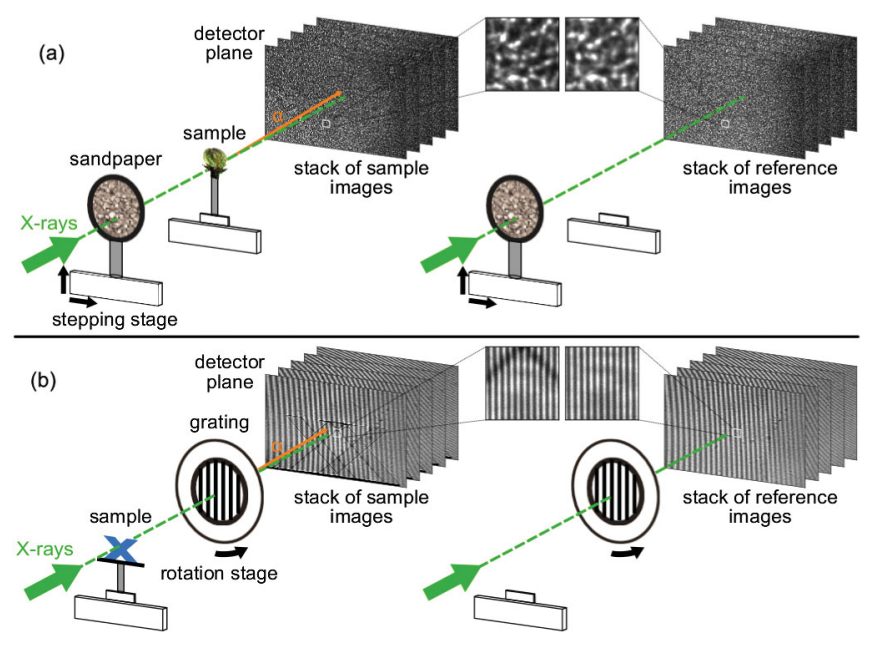

FIG. 1. Experimental setup using (a) a piece of abrasive paper and (b) a beam-splitter phase grating as a phase modulator creating a near-field interference pattern in the detector plane. The insets show $150 \times 150$ pixel regions of the patterns with (left) and without (right) the sample in the beam.

periodic or random. In the following, we characterize the performance of the technique for imaging and metrology experiments and demonstrate its flexibility to tune image quality and scan time.

A sketch of the experimental setup is shown in Fig. 1. Experiments were carried out at the I13-1 beamline at Diamond Light Source [36] with a $19-\mathrm{keV}$ x-ray beam selected by a horizontally deflecting monochromator. A near-field interference pattern is created in the detector plane by a phase modulator (PM): a near-field speckle pattern produced by a piece of abrasive paper (combination of granularities P800 and P5000 [37]) in Fig. 1(a) or a line interference pattern from a beam-splitter phase grating (material: SU-8 photoresist polymer, period: $5.4 \mu \mathrm{m}$, line height: $26.7 \mu \mathrm{m}$, fabricated by IMT-KIT, Germany) in Fig. 1(b). Projections with and without the sample in the beam were recorded for different positions of the PM. Each projection was exposed for $4 \mathrm{~s}$. A pco4000 CCD camera (pixel size: $9 \mu \mathrm{m}$ ) coupled to magnifying optics and a scintillation screen was used, providing an effective pixel size of $p_{\text {eff }}=0.40 \mu \mathrm{m}$. The sandpaper was placed $0.17 \mathrm{~m}$ upstream and the detector $0.34 \mathrm{~m}$ downstream of the sample. The grating was located $0.16 \mathrm{~m}$ downstream of the sample [38] and the detector was placed another $0.18 \mathrm{~m}$ from the grating close to the first fractional Talbot distance [39]. The sandpaper was scanned on a spiral pattern and images were recorded at 24 PM positions with step sizes of about $28 \mu \mathrm{m}$ - larger than the average speckle size. The grating was rotated around the optical axis from $0^{\circ}$ to $90^{\circ}$ in increments of $2^{\circ}$ between each of the 46 projections.

The modulation of the reference pattern caused by the sample used in the UMPA reconstruction approach has been described previously in Ref. [21]. According to this model, the intensity $I_{j}(\mathbf{r})$ of the $j$ th out of $N$ interference patterns can be expressed in terms of the undisturbed pattern $I_{0 j}(\mathbf{r})$ as follows:

$$
\begin{aligned}
I_{j}(\mathbf{r}) & =T(\mathbf{r})\left\{\langle I\rangle+v(\mathbf{r})\left[I_{0 j}(\mathbf{r}+\mathbf{u})-\langle I\rangle\right]\right\} \\
& =\beta(\mathbf{r})\langle I\rangle+\kappa(\mathbf{r}) I_{0 j}(\mathbf{r}+\mathbf{u}),
\end{aligned}
$$

where $T(\mathbf{r})$ is the sample's transmission, $v(\mathbf{r})$ is the visibility related to small-angle scattering, and $\mathbf{u}$ is a displacement vector proportional to the refraction angle. $\langle I\rangle$ is the mean intensity of the reference pattern and we define $\beta=T(1-v)$ and $\kappa=T v$. A least-square formulation of the problem allows us to find these quantities from the set of measurements, by minimizing the cost function:

$\mathcal{L}=\sum_{\mathbf{r}} \sum_{j}^{N} \Gamma\left(\mathbf{r}-\mathbf{r}_{0}\right)\left|I_{j}(\mathbf{r})-\beta(\mathbf{r})\langle I\rangle-\kappa(\mathbf{r}) I_{0 j}(\mathbf{r}+\mathbf{u})\right|^{2}$.

Here, $\Gamma$ is a normalized window function centered at position $\mathbf{r}_{0}$, whose width influences both the sensitivity of the reconstructed refraction angle signal and the spatial resolution. Minimization of Eq. (2) with respect to $\beta, \kappa$, and $\mathbf{u}$ for all window centers $\mathbf{r}_{0}$ yields the four independent signal maps $T, v$, and $\mathbf{u}$. The refraction angle $\left(\alpha_{x}, \alpha_{y}\right)$ and the phase gradient $(\partial \Phi / \partial x, \partial \Phi / \partial y)$ are obtained from $\mathbf{u}$ through simple geometric transformations (under smallangle approximation),

$$
\left(\frac{\partial \Phi}{\partial x}, \frac{\partial \Phi}{\partial y}\right)=\frac{2 \pi}{\lambda}\left(\alpha_{x}, \alpha_{y}\right)=\frac{2 \pi}{\lambda}\left(u_{x}, u_{y}\right) \frac{p_{\mathrm{eff}}}{d},
$$

where $d$ is the propagation distance to the detector. The total phase shift $\Phi$ experienced by the x-rays passing through the sample is then recovered via 2D Fourier integration of the differential phase signals $[15,40]$.

In a previous work, a similar method was used to obtain these quantities from a single speckle measurement [21]. However, for the problem to be well behaved one had to set the width of $\Gamma$ larger than the speckle size as smaller windows do not track speckles reliably. The approach with a single measurement also required diversity in both the $x$ and $y$ direction, ruling out its application with linear gratings. Combining multiple measurements in a single reconstruction lifts these limitations. It can be shown that the model of Eq. (2) also encompasses the speckle-scanning scheme, by letting the window reduce to a single-pixel box function.

As a first demonstration the UMPA technique with a random PM was applied to a biological sample, a small flower bud (Cotoneaster dammeri radicans). The visibility, defined as the ratio of standard deviation and mean intensity of the speckle pattern in a $150 \times 150$ pixels region without sample, was on average $43 \%$ in the left half of the field of view and $37 \%$ in the right part, with the 

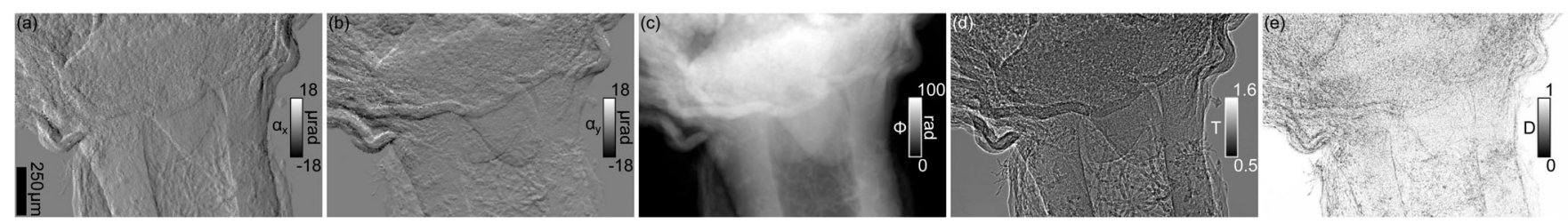

FIG. 2. Multimodal images of a small flower bud (Cotoneaster dammeri radicans) imaged with the speckle setup and reconstructed with $N=24$ and $w=5$. (a) Refraction in the horizontal direction, (b) refraction in the vertical direction, (c) phase shift, (d) transmission, and (e) dark-field signal.

difference due to slightly slanted mounting of the scintillation screen. The size of the speckles determined via 2D autocorrelation analysis of the speckle pattern as described in Ref. [41] was approximately 34 pixels $\approx 13.7 \mu \mathrm{m}$ in the horizontal and 28 pixels $\approx 11.3 \mu \mathrm{m}$ in the vertical direction. This asymmetry is explained by a smaller transverse coherence in the horizontal direction. The reconstruction of the differential phase, transmission, and dark-field signals was performed using an analysis window of size $w \times w=5 \times 5$ pixels [normalized Hamming window with a full width at half maximum (FWHM) of 2 pixels]. The corresponding images are presented in Fig. 2. The differential phase signals in the horizontal and vertical directions in Figs. 2(a) and 2(b), respectively, reveal the detailed inner structure of the flower bud. The petals in the upper part show a granular texture. In the bottom part, the vertical walls of the stalk are clearly visible, in particular in the horizontal refraction angle in Fig. 2(a), and a complex tube network can be observed inside the stalk. This fine tubular composition and a strong phase shift in the upper part of the sample are visible in the integrated phase signal in Fig. 2(c). While the transmission image in Fig. 2(d) mainly highlights the outlines of the features due to edge enhancement effects occurring upon propagation, the dark field in Fig. 2(e) reveals strong scattering from the small granular features, especially in the upper part of the specimen.

The spatial resolution is not limited by the speckle size. Its ideal value is equal to twice the FWHM of the window, giving in the present case a resolution of 4 pixels $\approx 1.6 \mu \mathrm{m}$. For small window sizes the achievable resolution reaches a limit imposed by experimental factors such as the point spread function of the detector. As a lower limit of the spatial resolution of the reconstructed phase image [42], we take the FWHM of two of the smallest discernible features in Fig. 2(c), which gives values of 4.8 and $5.1 \mu \mathrm{m}$. This estimate is consistent with data from a test pattern [43]. The angular sensitivity of the measurement-evaluated by computing the standard deviation of the refraction angle signal in a region of $150 \times 150$ pixels in the background area of the image - is $79 \mathrm{nrad}$ in the horizontal and $66 \mathrm{nrad}$ in the vertical direction. The sensitivity is better in the vertical due to the higher transverse coherence of the x-ray beam in this direction.

As in many other imaging systems, sensitivity and resolution are not independent quantities. Performing error propagation from the raw measurements to the reconstructed refraction maps, one finds that the variance $\sigma^{2}$ in the signal is inversely proportional to $N w^{2}$, the number of independent contributions to the extraction of the signal in one pixel. Hence, the angular sensitivity $\sigma$, defined as the standard deviation of the refraction angle signal, can be expressed by the relation

$$
\sigma=\frac{C}{w \sqrt{N}},
$$

where $C$ is a constant depending on the measurement conditions. This relation, already observed for photographic emulsions many decades ago [46], generally applies to imaging systems whose noise is devoid of spatial correlations. While Eq. (4) does not encompass all experimental contributions to the signal error, such as photon counting statistics, readout noise, and PM position errors as discussed, e.g., in Refs. [47,48], it confirms the validity and tunable character of our reconstruction method.

Relation (4) explains how to beat the resolution limit imposed by the feature size of the reference pattern: to decrease $w$ while keeping the same sensitivity $\sigma$, one simply needs to include more independent measurements $N$. Figure 3 illustrates this possibility with experimental data. A sample made from polymethyl methacrylate spheres of diameters up to $80 \mu \mathrm{m}$ and polystyrene spheres of diameter $250-350 \mu \mathrm{m}$ in a polyvinyl chloride tube was imaged with the setup in Fig. 1 using a random PM. Figure 3(a) shows the refraction angle signal $\alpha_{x}$ with the parameters $N=16$ and $w=21$. A region of interest $(\mathrm{ROI})$ of $370 \times 370$ pixels-as indicated by the red box-is shown in Fig. 3(b). The relationship between the quantities in Eq. (4) is visualized for a few fixed $w$ and $N$ values in the line plots in Figs. 3(i) and 3(j), respectively. The constant $C=2236$ nrad was determined from a weighted least-square fit of the experimental $\sigma$ values to Eq. (4) [49]. The measured $\sigma$ for selected parameter pairs $N, w$ are plotted as colored dots in Figs. 3(i) and $3(\mathrm{j})$ and they agree well with the theoretical model. For these parameters, the corresponding ROIs are presented in Figs. 3(c)-3(h). The gain in angular sensitivity can clearly be observed for increasing $N$, see Figs. 3(c)-3(e), and larger window sizes $w$, see Figs. 3(f)-3(h), as predicted by Eq. (4). The latter, however, also leads to a decrease in spatial resolution. For $N=1$, equivalent to single-shot $\mathrm{SBI}$, in Fig. 3(c), the sparsity of information gives rise to systematic errors at the edges of the sphere. 

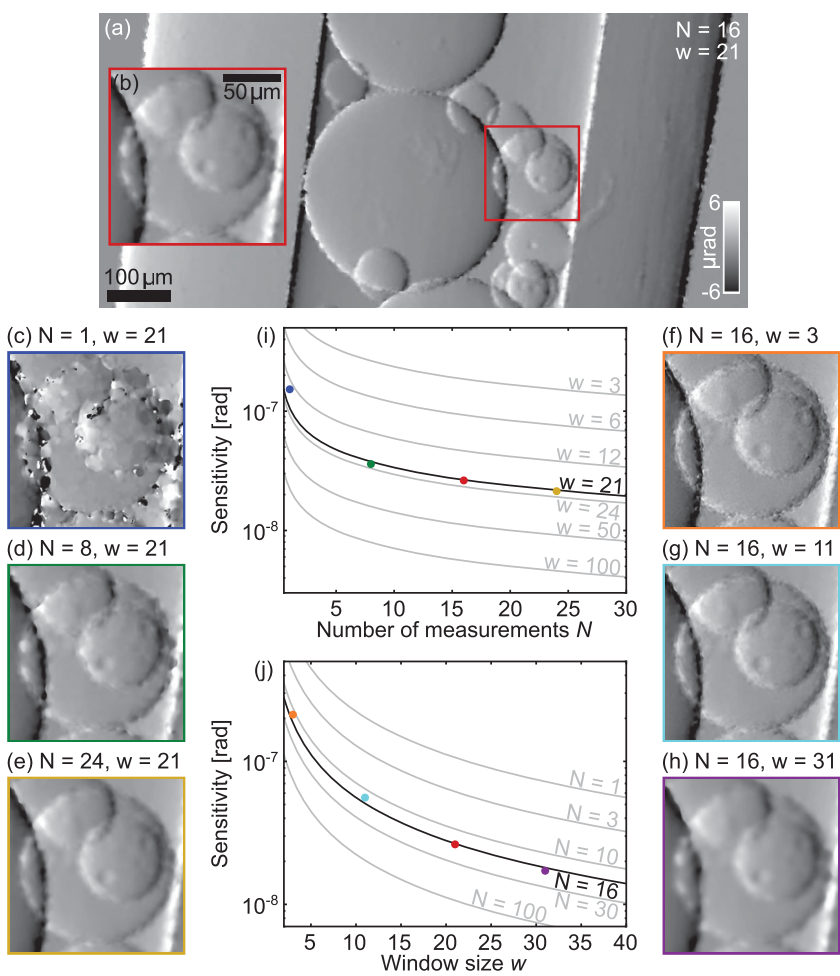

FIG. 3. (a) Refraction $\alpha_{x}$ in the horizontal direction $\alpha_{x}$ of a sphere sample imaged using a sandpaper diffuser and reconstructed with $N=16$ and $w=21$ and (b) region of interest $(370 \times 370$ pixels $)$. (c)-(h) Same regions reconstructed with different parameter combinations $N, w$. (i)-(j) Relationship between angular sensitivity $\sigma$ and number of measurements $N$ or analysis window size $w$, respectively, as described by Eq. (4). Experimentally measured values are plotted as colored dots and agree well with the model.

The resolution-sensitivity relation given by Eq. (4) holds true also when the reference pattern is periodic. Here, we use the grating setup described above to perform metrology on a polymer (SU-8 photoresist) point-focus compound refractive lens (CRL) [50] fabricated at IMT-KIT, Germany. The aim of this measurement was to evaluate beam damage from extreme $\mathrm{x}$-ray exposure during previous use as an optics element [51]. The period of the grating interference pattern, measured by fitting the line pattern to a sinusoidal curve, was approximately 13.5 pixels $\approx 5.4 \mu \mathrm{m}$. The mean visibility was determined from the same fit as $\left(I_{\max }-I_{\min }\right) /$ $\left(I_{\max }+I_{\min }\right)$, where $I_{\min }$ and $I_{\max }$ are the minimum and maximum intensities of the fit curve, respectively. The visibility was on average $30.4 \%$ in the left half and $23.4 \%$ in the right half of the field of view. The angular sensitivities for this setup were $\sigma_{x}=114 \mathrm{nrad}$, $\sigma_{y}=94 \mathrm{nrad}$. The refraction signals reconstructed with a window size $w=5$ and the integrated phase are shown in Figs. 4(a)-4(c). The line profiles through the center of the differential phase signals in the horizontal (blue) and the vertical direction (red) in Fig. 4(d) and through the
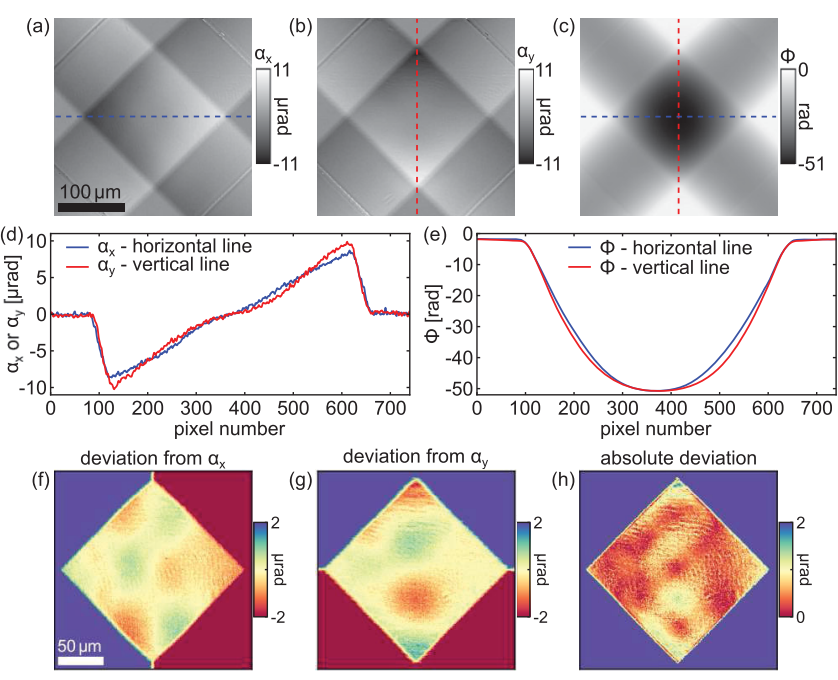

FIG. 4. Refraction signals in (a) the horizontal and (b) the vertical direction of a polymer compound refractive lens measured with a periodic line reference pattern and reconstructed with $N=46$ and $w=5$ and (c) integrated phase shift. Line profiles through the center of the lens for (d) the refraction signals and (e) the phase signal. (f) $-(\mathrm{g})$ Residuals from a linear fit to the refraction angle in the two directions and (h) absolute magnitude of the combined deviation vector.

integrated phase shift in Fig. 4(e) reveal an asymmetry in the refraction properties of the lens. This observation is confirmed by the residuals from a linear fit to the refraction angle [52] in the central focusing part of the lens shown in Figs. 4(f) and 4(g). Figure 4(h) is the magnitude of the combined deviation vector visualizing the absolute deviation from the expected refraction behavior of the lens, which is about 2 orders of magnitude larger than for CRLs without beam damage [52].

In this Letter we have demonstrated that UMPA is a tunable and versatile method for $\mathrm{x}$-ray phase-sensitive imaging and metrology. It provides an elegant solution to the main limitations of both $\mathrm{x}$-ray grating interferometry and speckle-based imaging. It lifts the need for perfect gratings and, when used with random phase modulators, decreases the number of frames by an order of magnitude with respect to previous implementations. By relaxing the requirements on the phase modulator structure and the scanning step size and precision, UMPA will accelerate the implementation of phase-contrast imaging setups, in particular at laboratory sources, thus also promoting the development of clinical applications [53]. The algorithm, of which our implementation is available for download [54], allows one to tune spatial resolution and angular sensitivity to adapt to the experimental constraints.

The proposed technique can be easily employed in the tomographic mode and we anticipate that it will find applications in a broad range of fields such as biomedical imaging and material characterization, as well as metrology 
and wave front sensing. We expect our approach to be quickly adopted for other wavelength regimes such as laser light and electron beams.

We thank K. Wanelik, S. Logan, X. Shi, and U. Wagner from Diamond Light Source and A. Wilson from Observatory Sciences Ltd. for their support in preparation and during the experiment. We thank N. Hirao and Y. Ohishi from Japan Synchrotron Radiation Institute for providing information about the previous dose exposure of the compound refractive lens. We acknowledge Diamond Light Source for beam time at the I13-1 beamline under proposal MT14562-1. We acknowledge funding from the European Research Council (FP7) Starting Grant No. 279753.

*marie-christine.zdora@diamond.ac.uk

${ }^{\dagger}$ Present address: Diamond Light Source, Harwell Science and Innovation Campus, Didcot, Oxfordshire OX11 ODE, United Kingdom.

[1] D. L. Misell, J. Phys. D 6, L6 (1973).

[2] P. F. Almoro and S. G. Hanson, Opt. Express 16, 7608 (2008).

[3] Y. Zhang, G. Pedrini, W. Osten, and H. J. Tiziani, Opt. Express 11, 3234 (2003).

[4] J. M. Rodenburg, A. C. Hurst, and A. G. Cullis, Ultramicroscopy 107, 227 (2007).

[5] S. W. Wilkins, T. E. Gureyev, D. Gao, A. Pogany, and A. W. Stevenson, Nature (London) 384, 335 (1996).

[6] P. Cloetens, W. Ludwig, J. Baruchel, D. Van Dyck, J. Van Landuyt, J. P. Guigay, and M. Schlenker, Appl. Phys. Lett. 75, 2912 (1999).

[7] J. M. Rodenburg, A. C. Hurst, A. G. Cullis, B. R. Dobson, F. Pfeiffer, O. Bunk, C. David, K. Jefimovs, and I. Johnson, Phys. Rev. Lett. 98, 034801 (2007).

[8] P. Thibault, M. Dierolf, A. Menzel, O. Bunk, C. David, and F. Pfeiffer, Science 321, 379 (2008).

[9] M. Stockmar, P. Cloetens, I. Zanette, B. Enders, M. Dierolf, F. Pfeiffer, and P. Thibault, Sci. Rep. 3, 1927 (2013).

[10] D. S. Acton, Proc. SPIE Int. Soc. Opt. Eng. 5487, 887 (2004).

[11] C. David, B. Nöhammer, H. H. Solak, and E. Ziegler, Appl. Phys. Lett. 81, 3287 (2002).

[12] A. Momose, S. Kawamoto, I. Koyama, Y. Hamaishi, K. Takai, and Y. Suzuki, Jpn. J. Appl. Phys. 42, L866 (2003).

[13] T. Weitkamp, A. Diaz, C. David, F. Pfeiffer, M. Stampanoni, P. Cloetens, and E. Ziegler, Appl. Phys. Lett. 86, 054101 (2005).

[14] S. Berujon, E. Ziegler, R. Cerbino, and L. Peverini, Phys. Rev. Lett. 108, 158102 (2012).

[15] K. S. Morgan, D. M. Paganin, and K. K. W. Siu, Appl. Phys. Lett. 100, 124102 (2012).

[16] S. Berujon, H. Wang, and K. Sawhney, Phys. Rev. A 86, 063813 (2012).

[17] F. Pfeiffer, M. Bech, O. Bunk, P. Kraft, E. F. Eikenberry, C. Brönnimann, C. Grünzweig, and C. David, Nat. Mater. 7, 134 (2008).

[18] W. Yashiro, Y. Terui, K. Kawabata, and A. Momose, Opt. Express 18, 16890 (2010).
[19] H. Wang, Y. Kashyap, and K. Sawhney, Phys. Rev. Lett. 114, 103901 (2015)

[20] F. Pfeiffer, T. Weitkamp, O. Bunk, and C. David, Nat. Phys. 2, 258 (2006).

[21] I. Zanette, T. Zhou, A. Burvall, U. Lundström, D. H. Larsson, M. Zdora, P. Thibault, F. Pfeiffer, and H. M. Hertz, Phys. Rev. Lett. 112, 253903 (2014).

[22] T. Zhou, I. Zanette, M. Zdora, U. Lundström, D. H. Larsson, H. M. Hertz, F. Pfeiffer, and A. Burvall, Opt. Lett. 40, 2822 (2015).

[23] T. Weitkamp, A. Diaz, C. David, F. Pfeiffer, M. Stampanoni, P. Cloetens, and E. Ziegler, Opt. Express 13, 6296 (2005).

[24] A. Momose, Jpn. J. Appl. Phys. 44, 6355 (2005).

[25] F. Pfeiffer, C. Kottler, O. Bunk, and C. David, Phys. Rev. Lett. 98, 108105 (2007).

[26] I. Zanette, M. Zdora, T. Zhou, A. Burvall, D. H. Larsson, P. Thibault, H. M. Hertz, and F. Pfeiffer, Proc. Natl. Acad. Sci. U.S.A. 112, 12569 (2015).

[27] H. Wang, S. Berujon, J. Herzen, R. Atwood, D. Laundy, A. Hipp, and K. Sawhney, Sci. Rep. 5, 8762 (2015).

[28] H. Wang, Y. Kashyap, and K. Sawhney, Appl. Phys. Lett. 108, 124102(2016).

[29] S. Berujon and E. Ziegler, Phys. Rev. Applied 5, 044014 (2016).

[30] N. Bevins, J. Zambelli, K. Li, Z. Qi, and G.-H. Chen, Med. Phys. 39, 424 (2012).

[31] H. Miao, L. Chen, E. E. Bennett, N. M. Adamo, A. A. Gomella, A. M. DeLuca, A. Patel, N. Y. Morgan, and H. Wen, Proc. Natl. Acad. Sci. U.S.A. 110, 19268 (2013).

[32] H. Wang, Y. Kashyap, and K. Sawhney, Sci. Rep. 6, 20476 (2016).

[33] S. Berujon and E. Ziegler, Phys. Rev. A 92, 013837 (2015).

[34] A. Hipp, J. Herzen, J. U. Hammel, P. Lytaev, A. Schreyer, and F. Beckmann, Proc. SPIE Int. Soc. Opt. Eng. 9967, 996718 (2016).

[35] M. Marschner, M. Willner, G. Potdevin, A. Fehringer, P. B. Noël, F. Pfeiffer, and J. Herzen, Sci. Rep. 6, 23953 (2016).

[36] C. Rau, U. Wagner, Z. Pesic, and A. D. Fanis, Phys. Status Solidi A 208, 2522 (2011).

[37] See FEPA P-grit classification for coated abrasives under http://www.fepa-abrasives.org/.

[38] The two PMs were placed at different positions relative to the sample due to mechanical limitations in the setup, which, however, does not affect the quantitative results of the measurements.

[39] P. Cloetens, J. P. Guigay, C. D. Martino, J. Baruchel, and M. Schlenker, Opt. Lett. 22, 1059 (1997).

[40] C. Kottler, C. David, F. Pfeiffer, and O. Bunk, Opt. Express 15, 1175 (2007).

[41] M.-C. Zdora, P. Thibault, F. Pfeiffer, and I. Zanette, J. Appl. Phys. 118, 113105 (2015).

[42] The spatial resolution of the phase image will in general be decreased compared to the differential phase signal due to the additional image processing step of $2 \mathrm{D}$ integration.

[43] See Supplemental Material at http://link.aps.org/ supplemental/10.1103/PhysRevLett.118.203903, which includes Refs. [44,45], for more details about the estimation of the spatial resolution.

[44] B. L. Henke, E. M. Gullikson, and J. C. Davis, At. Data Nucl. Data Tables 54, 181 (1993). 
[45] I. Zanette, T. Weitkamp, T. Donath, S. Rutishauser, and C. David, Phys. Rev. Lett. 105, 248102 (2010).

[46] E. W. H. Selwyn, Photogr. J. 75, 571 (1935).

[47] W. Yashiro, Y. Takeda, and A. Momose, J. Opt. Soc. Am. A 25, 2025 (2008).

[48] V. Revol, C. Kottler, R. Kaufmann, U. Straumann, and C. Urban, Rev. Sci. Instrum. 81, 073709 (2010).

[49] See Supplemental Material at http://link.aps.org/ supplemental/10.1103/PhysRevLett.118.203903, which includes Refs. [44,45], for more details on the fit and validation of the UMPA algorithm.

[50] V. Nazmov, E. Reznikova, J. Mohr, A. Snigirev, I. Snigireva, S. Achenbach, and V. Saile, Microsyst. Technol. 10, 716 (2004).
[51] The lens was repeatedly exposed to x-rays from an undulator synchrotron source at the SPring-8, BL10XU beamline with prefocus-lens ( $2 \mathrm{~mm}$ aperture beryllium lens) focusing the beam on a spot of approximately $50 \mu \mathrm{m}$. These extreme conditions led to heating and dose-induced damage of the polymer.

[52] F. J. Koch, C. Detlefs, T. J. Schröter, D. Kunka, A. Last, and J. Mohr, Opt. Express 24, 9168 (2016).

[53] J. Tanaka, M. Nagashima, K. Kido, Y. Hoshino, J. Kiyohara, C. Makifuchi, S. Nishino, S. Nagatsuka, and A. Momose, Z. Med. Phys. 23, 222 (2013).

[54] See https://github.com/pierrethibault/UMPA. 